\title{
Estado del arte del análisis de gases disueltos en transformadores de potencia
}

\author{
State of the Art of Dissolved Gas Analysis in Power Transformers
}

Fecha de Recepción: 28 de Diciembre de 2013

Fecha de Aprobación: 28 de Enero de 2014
Johana Tatiana Sarria-Arias* Natalia Andrea Guerrero-Bello* Edwin Rivas-Trujillo**

\section{Resumen}

Presenta el estado del arte de las técnicas Dornenburg, Gases Claves, Rogers y Duval, las más empleadas para evaluar la condición del aislamiento de los transformadores de potencia; técnicas basadas en el análisis de gases disueltos en el aceite (DGA) e interpretación de resultados.

Palabras clave: Dornenburg, Gases Claves, Rogers, Duval, Transformadores de potencia, DGA

\section{Abstract}

This paper presents the state of the art, concerning the techniques (Dornenburg, Gas Key, Rogers, Duval) which are most used for insulation condition assessment of power transformers, based on dissolved gas analysis in oil (DGA).

Keywords: Dornenburg, Key Gases, Rogers, Duval, Power Transformers, DGA.

\footnotetext{
* Universidad Distrital “Francisco José de Caldas” (Bogotá-Cundinamarca, Colombia). johanatatiana@hotmail.com

** Universidad Distrital "Francisco José de Caldas" (Bogotá-Cundinamarca, Colombia). nataliaguerrerobello@hotmail.com

*** Universidad Distrital "Francisco José de Caldas” (Bogotá-Cundinamarca, Colombia). erivas@udistrital.edu.co
} 


\section{INTRODUCCIÓN}

Los materiales aislantes en transformadores se descomponen liberando gases dentro de la cuba; la distribución de estos gases puede estar relacionada con el tipo de avería del suministro eléctrico, y la tasa de generación de gas puede indicar su severidad. Existen varias técnicas para el mantenimiento y el diagnóstico de averías en transformadores de potencia en funcionamiento, entre ellas, el análisis de gases disueltos (DGA). El DGA es un método popular para diagnosticar e interpretar los diferentes tipos de averías en transformadores de potencia; para refinar las interpretaciones en el proceso de diagnóstico, actualmente se cuenta con técnicas como sistemas expertos y análisis de datos utilizando redes neuronales artificiales.

\section{EsTAdo DEL ARTE}

El análisis del diagnóstico de gases disueltos (DGA) inicia en 1960 y es ampliamente utilizado en el mundo para detectar averías incipientes en el transformador; en la Fig. 1 se muestra un diagrama conceptual de la aplicabilidad de este método, y en la Tabla 1 se plasman los trabajos más representativos con él reportados en la literatura científica.

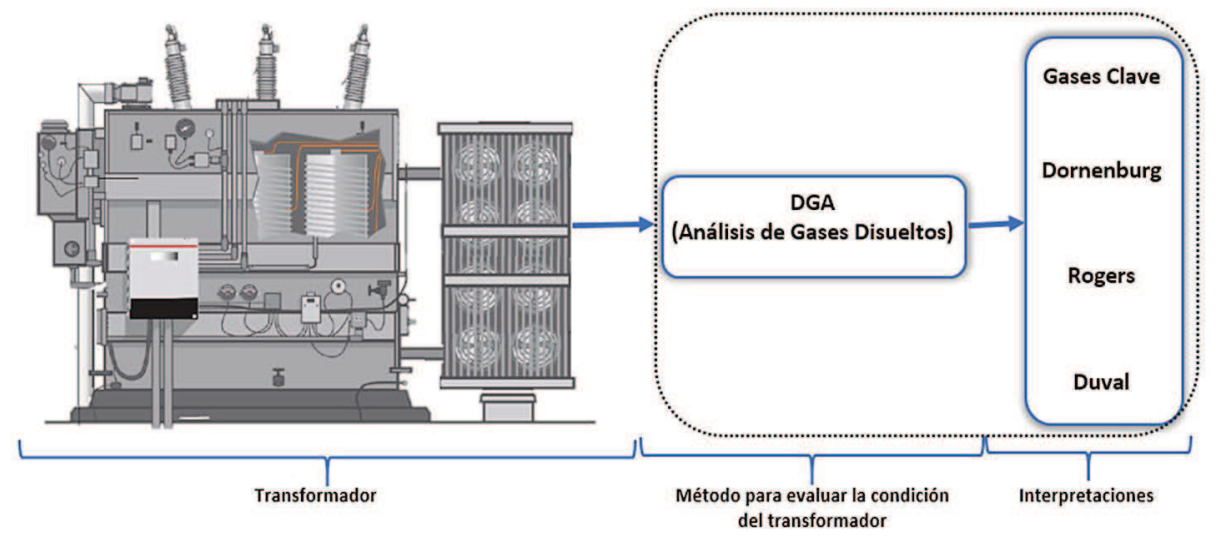

Fig. 1. Diagrama conceptual de la aplicabilidad del DGA

TABLA 1

TRABAJOS MÁS REPRESENTATIVOS REFERENTES A DGA REPORTADOS EN LA LITERATURA CIENTÍFICA

\begin{tabular}{|c|l|}
\hline Autores y año & \multicolumn{1}{c|}{ Aspectos relevantes } \\
\hline Michel Duval & $\begin{array}{l}\text { Simula averías en el laboratorio de DGA y presenta los resultados en forma } \\
\text { gráfica [1]. }\end{array}$ \\
\hline Sayed A. Ward & $\begin{array}{l}\text { Implementa DGA como herramienta de diagnóstico para evaluar la condición } \\
\text { de transformadores en servicio [2]. }\end{array}$ \\
\hline Michel Duval, James Dukarm & $\begin{array}{l}\text { A partir de datos de laboratorio del CIGRE propone que mejorar la evaluación } \\
\text { del diagnóstico del estado del transformador [3]. }\end{array}$ \\
\hline
\end{tabular}




\begin{tabular}{|c|l|}
\hline Autores y año & \multicolumn{1}{|c|}{ Aspectos relevantes } \\
\hline $\begin{array}{c}\text { Jl. Ganesha } \\
2006\end{array}$ & $\begin{array}{l}\text { Reporta datos experimentales de los efectos del arco en el gas disuelto en los } \\
\text { aceites del transformador [4]. }\end{array}$ \\
\hline $\begin{array}{c}\text { Michael Duval } \\
2008\end{array}$ & $\begin{array}{l}\text { Propone nuevas versiones de su triángulo clásico utilizando triángulo de Duval } \\
\text { para aceite mineral, triángulo 2 para cambiadores de toma de carga, triángulo } \\
3 \text { para aceites no minerales (esteres naturales o sintéticos) y finalmente los } \\
\text { triángulos 4 y 5 para averías de baja temperatura, donde la gasificación de } \\
\text { dispersión de aceites puede interferir con el diagnóstico [5]. }\end{array}$ \\
\hline $\begin{array}{c}\text { A. Akbari, A. Setayeshmehr, H. } \\
\text { Borsi, E. Gockenbach }\end{array}$ & $\begin{array}{l}\text { Diseñan una aplicación detallada de la técnica de Duval para los investigadores } \\
\text { y empresas de servicios públicos interesados en la visualización de sus propios } \\
\text { resultados del DGA con un programa de software [6]. }\end{array}$ \\
\hline $\begin{array}{c}\text { E. A. Mackenzie, J. Crossey, A. de } \\
\text { Pablo, W. Ferguson }\end{array}$ & $\begin{array}{l}\text { Muestra cómo el DGA puede ser utilizado para monitoreo en línea (on line) } \\
\text { detectando un 70\% de las averías más comunes en transformadores de potencia } \\
{[7] .}\end{array}$ \\
\hline $\begin{array}{c}\text { Mohammad Golkhah, Sahar } \\
\text { Shamshirgar Saffar y Mohammad } \\
\text { Ali Vahidi } \\
\text { 2011 }\end{array}$ & $\begin{array}{l}\text { Realizan la interpretación de diagnósticos de averías en transformadores de } \\
\text { potencia con base en redes neuronales artificiales (RNA) [8]. }\end{array}$ \\
\hline $\begin{array}{c}\text { Analiza y compara las siete técnicas de análisis de datos de gases disueltos más } \\
\text { comunes (Gas Key, Dornenburg Relación, Relación de Rogers, ábaco, Relación } \\
\text { de la CEI, Triángulo de Duval, y CIGRE) para interpretar el tipo de avería [9]. }\end{array}$
\end{tabular}

\section{A. Técnica de Dornenburg}

En 1970, Dornenburg propone una técnica para diferenciar el origen térmico o eléctrico de las averías; esta se basa en las cuatro relaciones entre concentraciones de gases (Ecuación 1).

$$
\begin{aligned}
& \boldsymbol{R} \mathbf{1}=\boldsymbol{R a z o ́ n} \mathbf{1}=\frac{\mathrm{CH}_{4}}{\mathrm{H}_{2}} ; \boldsymbol{R} 2=\boldsymbol{R a z o ́ n} \mathbf{2}=\frac{C_{2} H_{2}}{C_{2} H_{4}} ; \\
& \boldsymbol{R} 3=\operatorname{Razón~} 3=\frac{C_{2} H_{2}}{C_{4}} ; \boldsymbol{R} 4=\boldsymbol{R a z o ́ n ~} 4=\frac{C_{2} H_{6}}{C_{2} H_{2}}
\end{aligned}
$$

Esta técnica se puede aplicar solo si existe una cantidad adecuada de gases disueltos en el aceite, lo que es una desventaja, dado que las relaciones que se obtienen de los análisis no se ajustan a la interpretación del origen de la avería (Tabla 2).

TABLA 2

INTERPRETACIÓN DEL ORIGEN DE LA AVERÍA DE ACUERDO A DORNENBURG

\begin{tabular}{|c|c|c|c|c|}
\hline Avería originada por & $\boldsymbol{R} \mathbf{1}$ & $\boldsymbol{R} 2$ & $\boldsymbol{R} 3$ & $\boldsymbol{R} 4$ \\
\hline Descomposición térmica & $>1.0$ & $<0.75$ & $<0.3$ & $>0.4$ \\
\hline Corona (PD de baja intensidad) & $<0.1$ & No es significativa & $<0.3$ & $>0.4$ \\
\hline Arco (PD de alta intensidad) & $>0.1 \mathrm{y}<1.0$ & $>0.75$ & $>0.3$ & $<0.4$ \\
\hline
\end{tabular}




\section{B. Técnica de Gases Claves}

La presencia de los gases combustibles depende de la temperatura presente en el aceite del transformador. Cuando se presenta una avería, la temperatura del transformador aumenta y se libera gran cantidad de gases combustibles en la cuba del transformador. La técnica de Gas Clave detecta las averías mediante la medición de los gases individuales en lugar de calcular las proporciones de gas. Esta técnica tuvo sus inicios en 1973 en los laboratorios de Doble [10], estableciéndose oficialmente en 1974 [11].

En la Tabla 3 se presenta un resumen de los criterios de diagnóstico de la técnica de gases claves.

TABLA 3

Criterios de Diagnóstico de la técnica de Gases Claves

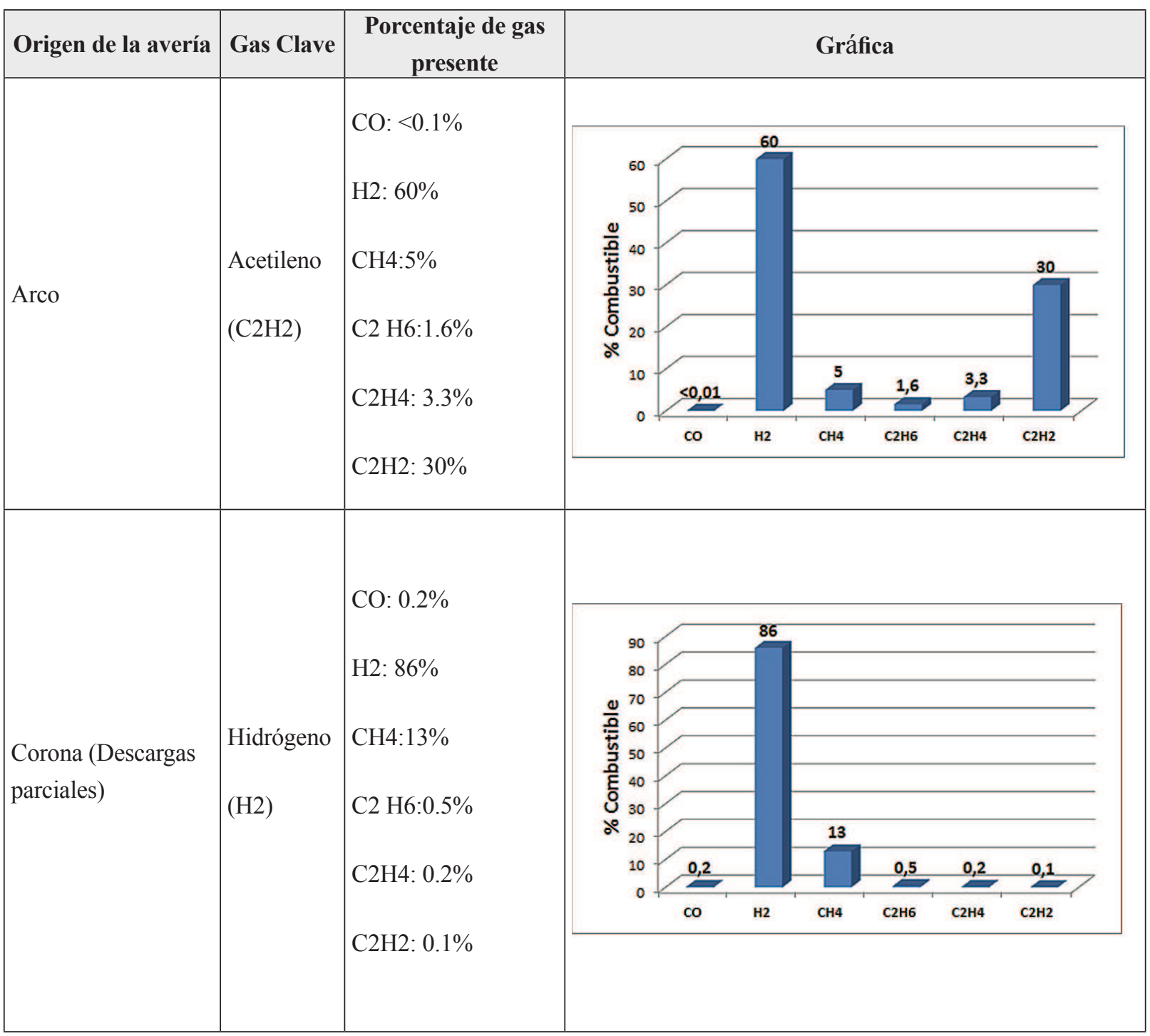




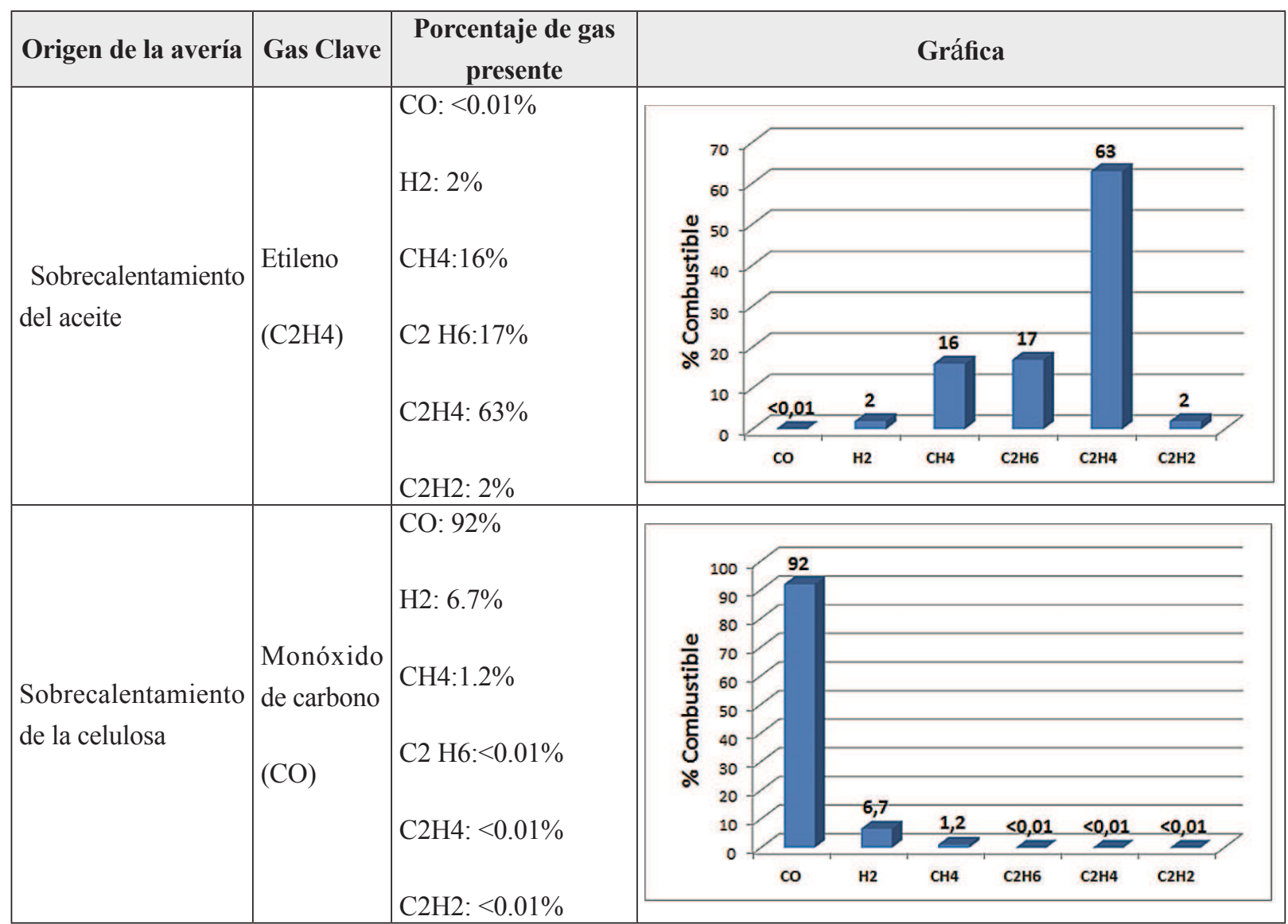

\section{Técnica de Rogers}

En 1978, Rogers observa que la concentración de cada gas varía con la temperatura de la avería, e introduce una nueva relación entre las concentraciones de los gases (Etileno y Acetileno) que requieren una temperatura más elevada para generarse. Concluye que el etano y el metano no ayudan en la identificación de la avería, y, por ende, las elimina de las relaciones utilizadas para esta técnica.

$$
\begin{aligned}
& \text { Razón 1 }=\frac{C H_{4}}{H_{2}} \\
& \text { Razón 2 }=\frac{C_{2} H_{2}}{C_{2} H_{4}} \\
& \text { Razón 3 }=\frac{C_{2} H_{4}}{C_{2} H_{6}}
\end{aligned}
$$

\section{Técnica de Duval}

En 1974, Duval [12] utiliza tres de los seis gases empleados por Dornenburg para clasificar averías en transformadores, y concluye que el hidrógeno se difunde más rápido en el metal que los otros gases hidrocarburos, distorsionando el diagnóstico.

En el 2002, Duval [1] interpreta los resultados del DGA en una forma gráfica y utiliza modelos de laboratorio para simular averías tomando como muestra ciento setenta y nueve casos reales (con el transformador en servicio), identificados por inspección visual, y diecinueve casos simulados en laboratorio.

Para la interpretación gráfica, Duval utiliza un triángulo equilátero compuesto por 3 vértices $\left(\% \mathrm{CH}_{4}, \% \mathrm{C}_{2} \mathrm{H}_{4} \mathrm{y} \% \mathrm{C}_{2} \mathrm{H}_{2}\right)$. Esta técnica (Ecuación 
3) propone un sistema de concentraciones en ppm (parte por millón)

$$
\begin{aligned}
& \% \boldsymbol{C}_{\mathbf{2}} \boldsymbol{H}_{2}=100 \frac{X}{X+Y+Z} \\
& \% \boldsymbol{C H}_{4}=100 \frac{Y}{X+Y+Z} \\
& \% \boldsymbol{C}_{2} \boldsymbol{H}_{4}=100 \frac{Z}{X+Y+Z}
\end{aligned}
$$

Siendo: X Concentración en ppm de $\mathrm{CH} 4, \mathrm{Y}$ concentración en ppm de $\mathrm{C} 2 \mathrm{H} 4 ; \mathrm{Z}$ concentración en ppm de $\mathrm{C} 2 \mathrm{H} 2$.

A manera de ejemplo, en la Fig. 2 se ilustra la forma de detectar una avería en el transformador. Las concentraciones $\mathrm{CH} 4, \mathrm{C} 2 \mathrm{H} 4$ y $\mathrm{C} 2 \mathrm{H} 2$ son los ejes coordenados del triángulo equilátero, donde se ha tomado $\mathrm{X}=\mathrm{Xp}=60 \mathrm{ppm}, \mathrm{Y}=\mathrm{Yp}=$ $30 \mathrm{ppm}$ y $\mathrm{Z}=\mathrm{Zp}=10 \mathrm{ppm}$. Se traza para $\mathrm{Xp}$ una recta paralela a $\mathrm{BC}$; para $\mathrm{Yp}$, una recta paralela a $\mathrm{CA}$, y para $\mathrm{Zp}$, una recta paralela a $\mathrm{AB}$, siendo la intersección de estas rectas el punto de ubicación de la avería.

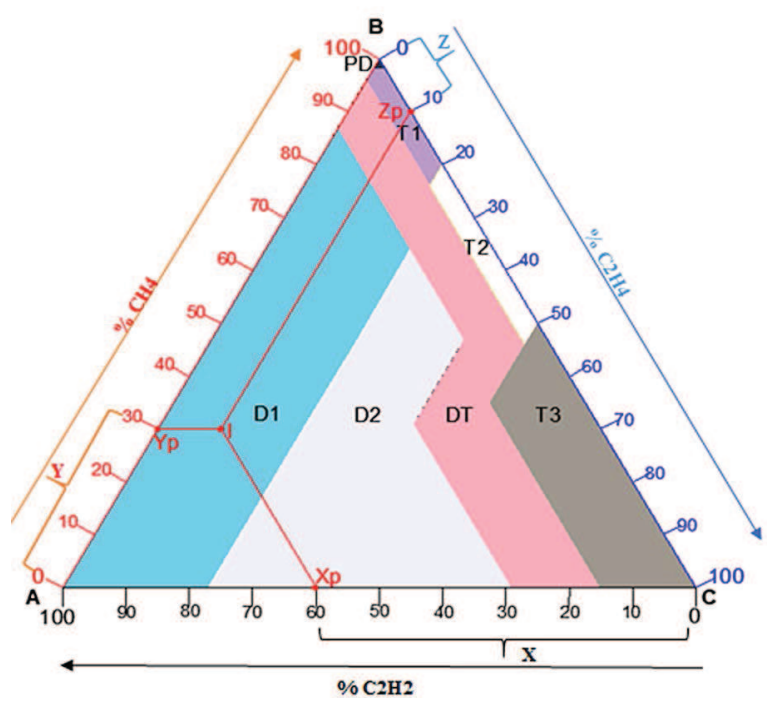

Fig. 2. Ubicación de avería en el triángulo de Duval

A partir de los casos analizados en laboratorio, Duval establece seis tipos de averías (PD, D1, D2, T1, T2, T3) detectables por el DGA (Tabla
4). Cuando el transformador se encuentra en servicio, la avería de T3 tiende a relacionarse con los puntos calientes en aceite, mientras que las averías T1 y T2, a puntos calientes en papel [1].

De acuerdo con el triángulo de Duval (Fig. 2), en la Tabla 4 se establecen los valores límites en ppm de los gases combustibles presentes en cada tipo de avería.

\section{TABLA 4}

DiAgNóstico DE TIPOS DE AVERÍA DEL Triángulo de Duval 1

\begin{tabular}{|l|l|l|}
\hline & \multicolumn{1}{|c|}{ Averías } & \multicolumn{1}{c|}{$\begin{array}{c}\text { Valores límites de gases } \\
\text { combustibles en porcentaje }\end{array}$} \\
\hline PD & Descargas parciales & $\mathrm{CH} 4=98$ \\
\hline D1 & $\begin{array}{l}\text { Descargas de baja } \\
\text { energía }\end{array}$ & $\mathrm{C} 2 \mathrm{H} 4=23-\mathrm{C} 2 \mathrm{H} 2=13$ \\
\hline D2 & $\begin{array}{l}\text { Descargas de alta } \\
\text { energía }\end{array}$ & $\mathrm{C} 2 \mathrm{H} 4=23 ; 40-\mathrm{C} 2 \mathrm{H} 2=13 ; 29$ \\
\hline T1 & $\begin{array}{l}\text { Averías térmicas a } \\
\mathrm{T}^{\circ}<300^{\circ} \mathrm{C}\end{array}$ & $\mathrm{CH} 4=98-\mathrm{C} 2 \mathrm{H} 4=20-\mathrm{C} 2 \mathrm{H} 2=4$ \\
\hline T2 & $\begin{array}{l}\text { Averías térmicas a } \\
300<\mathrm{T}^{\circ}<700^{\circ} \mathrm{C}\end{array}$ & $\mathrm{C} 2 \mathrm{H} 4=20-\mathrm{C} 2 \mathrm{H} 4=50-\mathrm{C} 2 \mathrm{H} 2=4$ \\
\hline T3 & $\begin{array}{l}\text { Averías térmicas a } \\
\mathrm{T}^{\circ}>700^{\circ} \mathrm{C}\end{array}$ & $\mathrm{C} 2 \mathrm{H} 4=50-\mathrm{C} 2 \mathrm{H} 2=15$ \\
\hline DT & $\begin{array}{l}\text { Mezcla de averías } \\
\text { térmicas y eléctricas }\end{array}$ & $\mathrm{C} 2 \mathrm{H} 2=13 ; 4 ; 29 ; 15-\mathrm{C} 2 \mathrm{H} 4=40,50$ \\
\hline
\end{tabular}

Michael Duval propuso nuevas versiones de su triángulo clásico [5], utilizando triángulo 1 para aceite mineral, triángulo 2 para cambiadores de toma en carga, triángulo 3 para aceites no minerales (esteres naturales o sintéticos) y, finalmente, triángulos 4 y 5 para averías de baja temperatura, donde la gasificación de dispersión de aceites puede interferir con el diagnóstico.

1) Estado del arte de la técnica de Michel Duval: Duval lleva a cabo un trabajo muy completo en el análisis de gases disueltos en el aceite (DGA) a partir del año 1974 hasta hoy, por ello es necesario hacer un análisis más detallado de su trabajo.

Como ya se anotó, Duval, en 1974 [12], utilizó tres de los seis gases empleados por Dornenburg para 
clasificar averías en transformadores, concluyendo que el hidrógeno se difunde más rápido en el metal que los otros gases hidrocarburos, lo cual distorsiona el diagnóstico. En 1989 [13] expone que los transformadores modernos de alta tensión están construidos con niveles de tolerancias de aislamiento relativamente bajos, siendo objeto de mayores tensiones en servicio comparados con transformadores antiguos, e indica que el DGA es una herramienta eficiente para el diagnóstico de transformadores en servicio con el fin de evitar averías, cortes y pérdidas de producción.

En 1990 [14] realizó un análisis de trazabilidad a 6046 transformadores en aceite de la compañía Hydro-Quebec; los análisis DGA se separaron en subgrupos de transformadores de potencia (5915 unidades) y distribución (131 unidades); adicionalmente, se separaron en función de la concentración de gas; concluyó que los transformadores de potencia son más propensos a la formación de puntos calientes y a averías producidas por puntos calientes, y menos susceptible a la formación de arcos eléctricos, así como a averías producidas por éstos.

En el 2001, Duval [15], con base en IEC 60599, referente a equipos eléctricos impregnados en aceite en servicio, la cual es una guía para la interpretación de los análisis de gases disueltos y libres, así como una base de datos de la IEC TC10 de equipos defectuosos inspeccionados en servicio, clasificó las averías de acuerdo con los principales tipos de errores que se pueden identificar mediante inspección visual; dentro de ellas se destacan las descargas parciales (PD) del tipo plasma frío (corona), con posible formación de cera y que provocan la inducción de pequeñas punciones carbonizadas en papel; las descargas de baja energía (D1), evidenciadas por perforaciones grandes en el papel o partículas de carbono en el aceite; las descargas de alta energía (D2), evidenciadas por carbonización; las averías térmicas por debajo de $300{ }^{\circ} \mathrm{C}$, si el papel se ha vuelto marrón (T1); por encima de $300{ }^{\circ} \mathrm{C}$, si el papel se ha carbonizado (T2); las averías térmicas por encima de $700{ }^{\circ} \mathrm{C}$ (T3), desplegado por la carbonización del aceite y la coloración del metal.

En el 2002, Duval [1] utiliza un método gráfico para interpretar los resultados del DGA; para ello utiliza modelos de laboratorio para simular resultados del DGA tomando como muestra 179 casos con el transformador en servicio, identificados por inspección visual, y 19 casos simulados en laboratorio; a partir de estos casos pudo establecer los seis tipos de averías principales PD, D1, D2, T1, T2, T3 (Tabla 4) y concluir que la avería $\mathrm{T} 3$ en servicio tiende a estar relacionada con puntos calientes en aceite, y que las averías T1 y T2 a puntos calientes en papel y la PD son potencialmente perjudiciales para el transformador.

En el 2006, Duval [12] hace una breve reseña sobre la interpretación del DGA en los transformadores, con un énfasis especial en la técnica del triángulo de Duval 1, y analiza cómo los resultados de laboratorio DGA pueden afectar a la fiabilidad del diagnóstico, ya que muy pocos laboratorios en el mundo proporcionan resultados precisos; por lo tanto, recomienda comprobar que los laboratorios cumplan con las normas; la precisión media de los laboratorios de todo el mundo ha sido evaluada por CIGRE TF11 como $\sim \pm 15 \%$.

En el 2008, Duval [16] propone varias técnicas para el cálculo de límites de gases individuales e intervalos de muestreo, con base en las recomendaciones y observaciones publicadas por CIGRE e IEC; y en diciembre de este mismo año expone las nuevas versiones del triángulo clásico de Duval [5], utilizando el triángulo 1 para aceite mineral, el triángulo 2 para cambiadores de toma de carga, el triángulo 3 para aceites no minerales (esteres naturales o sintéticos) y, finalmente, los triángulos 4 y 5 para averías de baja temperatura donde la gasificación de dispersión de aceites puede interferir con el diagnóstico (Tabla 6). 
Tabla 5

TriÁngulos de Duval, NúMeros 1 AL 5

\begin{tabular}{|l|l|l|}
\hline \multicolumn{1}{|c|}{ Descripción } \\
\hline 1 & Para transformadores con aceite mineral. Tiene 7 zonas (Tabla 4). \\
Los gases que toma son: \\
Cateto $\mathrm{X}=$ Acetileno (C2H2), \\
Cateto $\mathrm{Y}=$ Metano (CH4), \\
Cateto Z $=$ Etileno (C2H4).
\end{tabular}




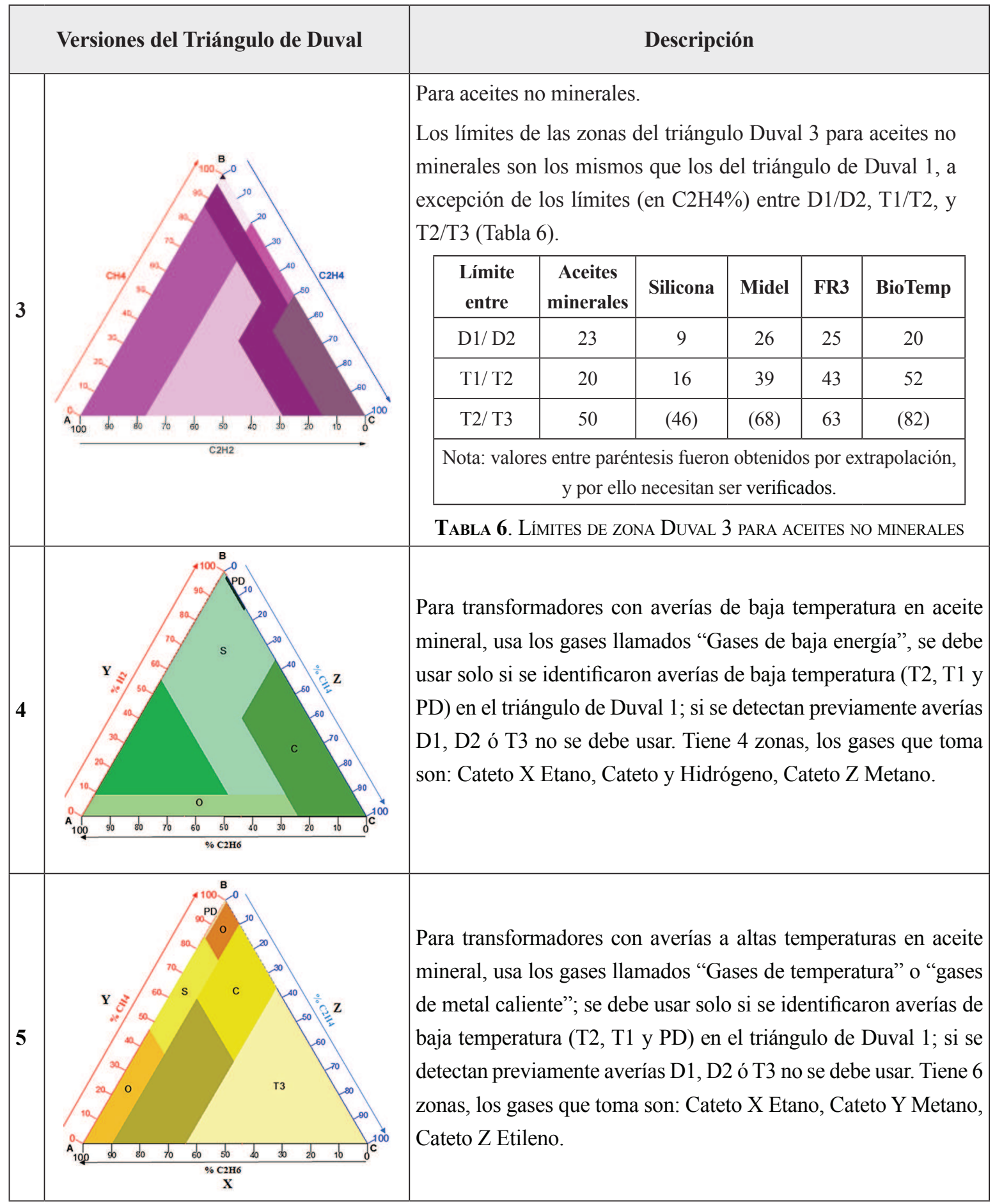


En el 2009, Duval [17] adiciona los triángulos 6 y 7 para las averías de baja temperatura en los transformadores con aceite dieléctrico vegetal biodegradable (FR3).

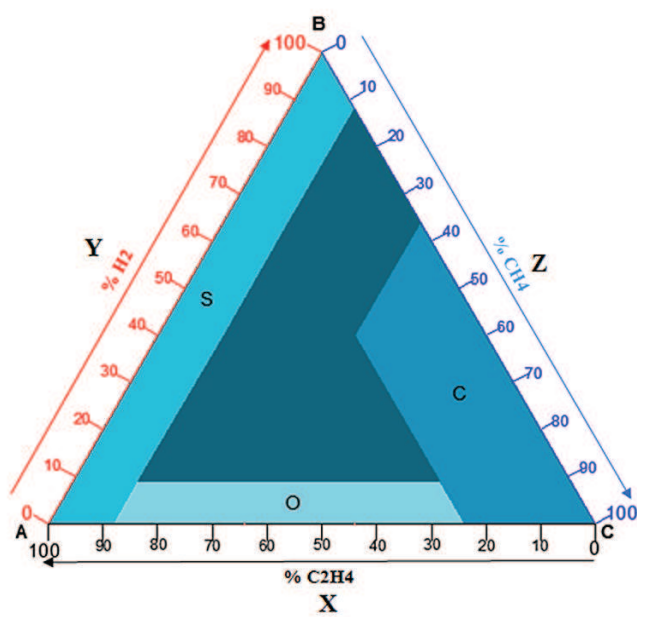

Fig. 3. El triángulo de Duval 6 para averías de baja temperatura en aceites FR3. Advertencia: los 3 gases utilizados en el triángulo 6 son $\mathrm{H} 2, \mathrm{CH} 4$ y C2H6

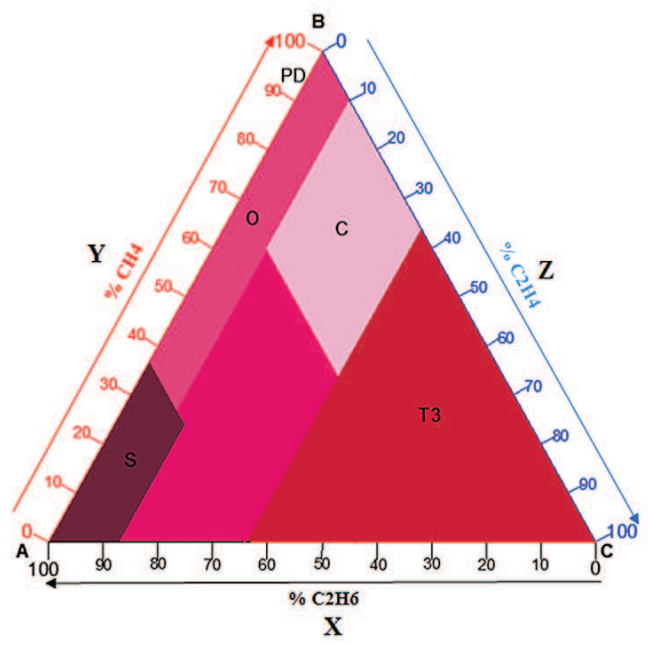

Fig. 4. El triángulo de Duval 7 para averías de baja temperatura en aceites FR3. Advertencia: los 3 gases utilizados en el triángulo 7 son $\mathrm{CH} 4, \mathrm{C} 2 \mathrm{H} 4 \mathrm{Y}$ C2H4.

En el 2012, Duval [18] presenta los triángulos 4 y 5 actualizados (Fig. 5 y Fig. 6). El triángulo de Duval 4 utiliza los gases $\mathrm{H} 2, \mathrm{CH} 4$ y $\mathrm{C} 2 \mathrm{H} 6$, que se forman más específicamente por averías de baja energía-temperatura (PD, T1 y T2); el triángulo 4 debe ser utilizado sólo para averías identificadas en primer lugar con el triángulo 1 , como las averías PD, T1 ó T2. El triángulo 5 debe ser utilizado solo para las averías identificadas en primer lugar con el triángulo 1 , como las averías T2 ó T3. Los triángulos 4 y 5 nunca se deben utilizar en caso de fallas D1 ó D2.

Las zonas de los triángulos indican: $\mathrm{S}=$ Pérdida de aceite a $\mathrm{T}<200{ }^{\circ} \mathrm{C}, \mathrm{O}=$ Sobrecalentamiento a $\mathrm{T}<250{ }^{\circ} \mathrm{C}$, pero no carbonizando el papel, $\mathrm{C}=$ posible carbonización del papel a $\mathrm{T}>300{ }^{\circ} \mathrm{C}$, $\mathrm{PD}=$ Corona descargas parciales, $\mathrm{T} 2=$ Averías térmicas $>300^{\circ} \mathrm{C}, \mathrm{T} 3=$ Averías térmicas $>700^{\circ} \mathrm{C}$.

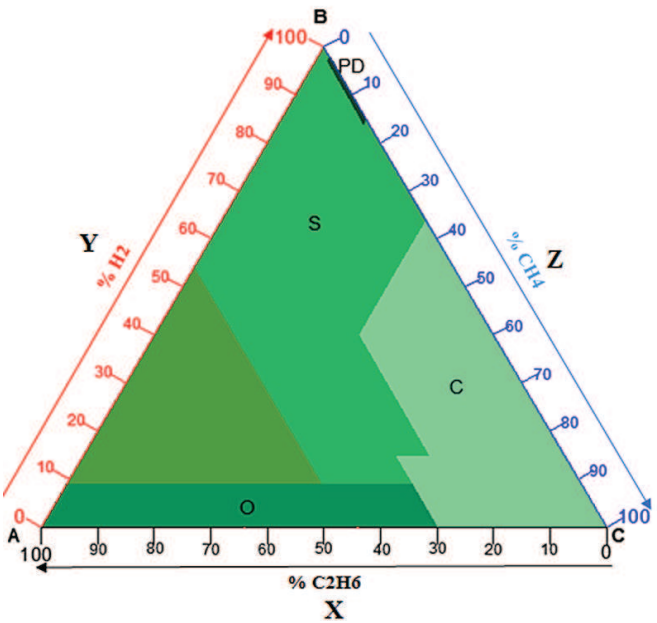

Fig. 5. Triángulo de Duval 4

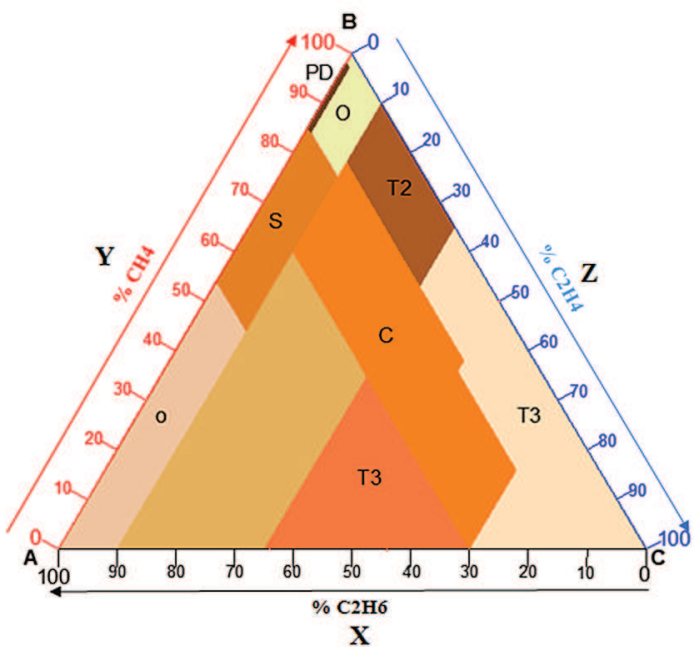

Fig. 6. Triángulo de Duval 5 
En el 2013, Duval [19] propone una nueva técnica de diagnóstico DGA, utilizando la IEC TC 10 y bases de datos relacionadas. Se analizaron cinco componentes de los gases (hidrógeno, metano, acetileno, etileno y etano), con el fin de determinar la clasificación de las averías claramente; las 5 razones de gases, R1-R5, usadas en técnicas de diagnóstico, como Doernenburg, Rogers y la IEC, suelen ser capaces de distinguir entre fallas térmicas (T1, T2 y T3, no está claramente dividido) y eléctricas (PD, D1 y D2); se pueden reorganizar en 10 razones de gases, como se observa en la Tabla 7. Hasta el momento, las razones R6-R10 no se han utilizado en las normas internacionales, pero logran distinguir averías de baja y alta temperatura entre averías térmicas.

\section{TABLA 7}

RESUltAdOS DEL ANÁLISIS DE LOS PATRONES DE AVERÍAS POR RAZONES DE GAS

\begin{tabular}{|c|c|c|c|c|c|}
\hline & N..$^{o}$ & Relaciones de Gas & $\begin{array}{c}\text { Entre averías } \\
\text { térmicas y eléctricas }\end{array}$ & $\begin{array}{c}\text { Entre averías } \\
\text { térmicas } \mathrm{T} 1 \sim \mathrm{T} 3 \\
\end{array}$ & $\begin{array}{c}\text { Entre averías eléctricas PD } \\
\text { D2 }\end{array}$ \\
\hline \multirow{5}{*}{$\begin{array}{l}\text { Relaciones } \\
\text { utilizadas en } \\
\text { técnicas de } \\
\text { diagnóstico }\end{array}$} & $\mathbf{R} 1$ & $\mathrm{CH} 4 / \mathrm{H} 2$ & ○ & $\mathrm{X}$ & O \\
\hline & $\mathbf{R 2}$ & $\mathrm{C} 2 \mathrm{H} 2 / \mathrm{C} 2 \mathrm{H} 4$ & O & $\mathrm{X}$ & $\boldsymbol{\Delta}$ \\
\hline & R3 & $\mathrm{C} 2 \mathrm{H} 2 / \mathrm{CH} 4$ & $\bigcirc$ & $\mathrm{X}$ & O \\
\hline & R4 & $\mathrm{C} 2 \mathrm{H} 6 / \mathrm{C} 2 \mathrm{H} 2$ & $\bigcirc$ & $\mathrm{X}$ & $\mathrm{O}$ \\
\hline & R5 & $\mathrm{C} 2 \mathrm{H} 4 / \mathrm{C} 2 \mathrm{H} 6$ & $\boldsymbol{\Delta}$ & $\Delta$ & O \\
\hline \multirow{6}{*}{$\begin{array}{l}\text { No se han usado } \\
\text { en normas } \\
\text { internacionales }\end{array}$} & R6 & $\mathrm{C} 2 \mathrm{H} 4 / \mathrm{CH} 4$ & O & O & $\mathrm{X}$ \\
\hline & $\mathbf{R} 7$ & $\mathrm{C} 2 \mathrm{H} 6 / \mathrm{CH} 4$ & $\mathrm{X}$ & $\mathrm{X}$ & $\mathrm{X}$ \\
\hline & R8 & $\mathrm{C} 2 \mathrm{H} 4 / \mathrm{H} 2$ & $\mathrm{X}$ & $\Delta$ & $\mathrm{X}$ \\
\hline & R9 & $\mathrm{C} 2 \mathrm{H} 6 / \mathrm{H} 2$ & $\Delta$ & $\mathrm{X}$ & $\mathrm{X}$ \\
\hline & R10 & $\mathrm{C} 2 \mathrm{H} 2 / \mathrm{H} 2$ & $\mathrm{X}$ & $\mathrm{X}$ & $\bigcirc$ \\
\hline & & O: Distinguible & $\Delta:$ Indefinido & \multicolumn{2}{|c|}{ X: Indistinguible } \\
\hline
\end{tabular}

\section{TABLA 8}

Clasificación DE AVERÍAS

A partir de los resultados del patrón de averías, las razones R1-R6 fueron clasificadas para 6 tipos de averías (Tabla 8), las razones seleccionadas se reorganizaron en 15 combinaciones (Tabla 9) de relación de gas para analizar los patrones de falla.

\begin{tabular}{|c|l|}
\hline Averías & \multicolumn{1}{|c|}{ Significado } \\
\hline PD & Descargas parciales corona \\
\hline D1 & Descarga de baja energía \\
\hline D2 & Descarga de alta energía \\
\hline T1 & Averías térmicas $\mathrm{T}<300^{\circ} \mathrm{C}$ \\
\hline T2 & Averías térmicas $300^{\circ} \mathrm{C}<\mathrm{T}<700^{\circ} \mathrm{C}$ \\
\hline T3 & Averías térmicas $\mathrm{T}>700^{\circ} \mathrm{C}$ \\
\hline
\end{tabular}


TABLA 9

RESULTADOS DE ANÁLISIS DE PATRONES FALLA CON COMBINACIÓN DE RAZÓN GAS

\begin{tabular}{|c|c|c|c|c|c|c|c|}
\hline \multirow{2}{*}{$\begin{array}{c}\text { Combinación de } \\
\text { relación gas }\end{array}$} & \multirow{2}{*}{$\begin{array}{c}\text { Distinción entre } \\
\text { averías eléctricas y } \\
\text { térmicas }\end{array}$} & \multicolumn{3}{|c|}{ Distinción entre fallas eléctricas } & \multicolumn{3}{|c|}{ Distinción entre fallas térmicas } \\
\hline & & $\boldsymbol{\Delta}$ & $\Delta$ & $\mathrm{X}$ & $\mathrm{X}$ & $\mathrm{X}$ & $\mathrm{X}$ \\
\hline R1-R3 & ○ & $\bullet$ & $\bullet$ & $\mathrm{X}$ & $\mathrm{X}$ & $\mathrm{X}$ & $\mathrm{X}$ \\
\hline R1-R4 & $\boldsymbol{\Delta}$ & $\bullet$ & $\bullet$ & $\mathrm{X}$ & $\mathrm{X}$ & $\mathrm{X}$ & $\mathrm{X}$ \\
\hline R1-R5 & $\Delta$ & $\bullet$ & $\bullet$ & $\mathrm{X}$ & $\Delta$ & $\circ$ & $\boldsymbol{\Delta}$ \\
\hline R1-R6 & $\boldsymbol{\Delta}$ & $\bullet$ & $\bullet$ & $\mathrm{X}$ & $\circ$ & $\bullet$ & ० \\
\hline R2-R3 & $\Delta$ & $\bullet$ & $\bullet$ & $\mathrm{X}$ & $\Delta$ & $\circ$ & $\boldsymbol{\Delta}$ \\
\hline R2-R4 & $\circ$ & $\bullet$ & $\bullet$ & $\mathrm{X}$ & $\boldsymbol{\Delta}$ & $\boldsymbol{\Delta}$ & $\mathrm{X}$ \\
\hline R2-R5 & $\circ$ & $\bullet$ & $\bullet$ & $\bullet$ & $\boldsymbol{\Delta}$ & ० & $\bullet$ \\
\hline R2-R6 & 0 & $\bullet$ & $\bullet$ & $\mathrm{X}$ & ○ & $\bullet$ & $\circ$ \\
\hline R3-R4 & $\mathrm{X}$ & $\bullet$ & $\bullet$ & $\mathrm{X}$ & $\mathrm{X}$ & $\mathrm{X}$ & $\mathrm{X}$ \\
\hline R3-R5 & $\mathrm{X}$ & $\bullet$ & $\bullet$ & $\mathrm{X}$ & $\boldsymbol{\Delta}$ & $\boldsymbol{\Delta}$ & $\boldsymbol{\Delta}$ \\
\hline R3-R6 & $\Delta$ & $\bullet$ & $\bullet$ & $\mathrm{X}$ & $\circ$ & $\bullet$ & $\circ$ \\
\hline R4-R5 & $\mathrm{X}$ & $\bullet$ & $\bullet$ & $\mathrm{X}$ & $\boldsymbol{\Delta}$ & $\boldsymbol{\Delta}$ & $\boldsymbol{\Delta}$ \\
\hline R4-R6 & $\mathrm{X}$ & $\bullet$ & $\bullet$ & $\mathrm{X}$ & ० & $\bullet$ & $\circ$ \\
\hline R5-R6 & $\mathrm{X}$ & $\bullet$ & $\bullet$ & $\mathrm{X}$ & $\bullet$ & $\bullet$ & $\circ$ \\
\hline •:Distinción clara & \multicolumn{3}{|c|}{ ○:Distinguible } & ndefi & \multicolumn{3}{|c|}{ X:Indistinguible } \\
\hline
\end{tabular}

La nueva técnica de diagnóstico clasifica cada avería en fases, mediante el usode 3 combinaciones de razones de gas (R1-R2, R2-R5 y R5-R6) (Tabla 9); la relación R1-R2 se utiliza para diferenciar entre averías térmicas y eléctricas, y la relación R5-R6, para identificar averías T1 de otras averías térmicas, para posteriormente identificar entre $\mathrm{T} 2$ y T3 mediante la relación R2-R5.

Se evaluaron razones de gas de cada tipo de avería mediante el uso de 122 casos del IEC TC 10 y bases de datos relacionadas (Figuras 7, 8 y 9); la precisión calculada para estos casos se muestra en la Tabla 10.

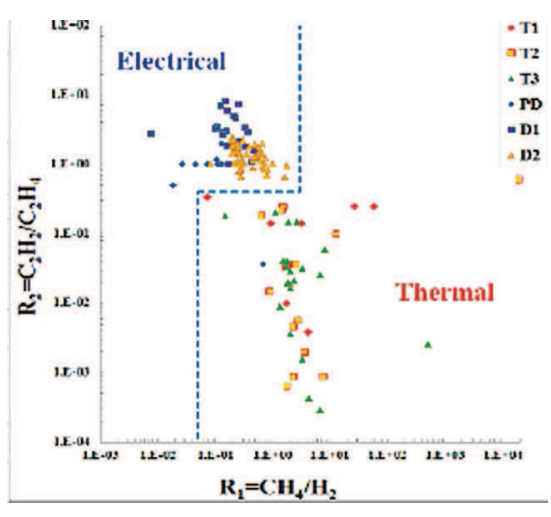

Fig. 7. Identificación entre las averías térmicas y eléctricas con la combinación de la relación de gas R1R2 


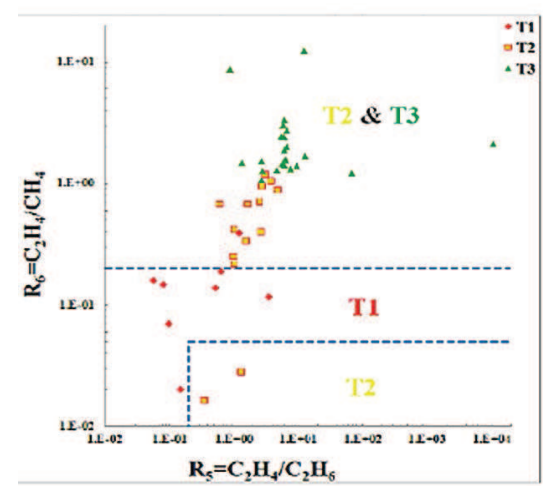

Fig. 8. Identificación entre las averías T1, T2 y T3 con combinación de la relación R5-R6

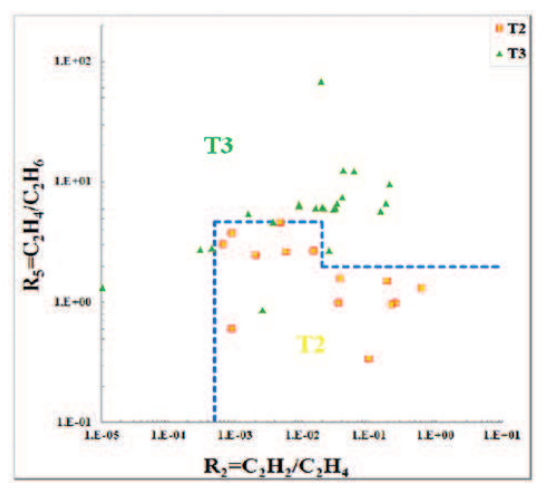

Fig. 9. Identificación entre las averías T2 y T3 con la combinación de la relación R2-R5

\section{TABLA 10}

COMPARACIÓN ENTRE LA NUEVA TÉCNICA Y LAS TÉCNICAS EXISTENTES

\begin{tabular}{|c|c|c|c|c|}
\hline Técnicas DGA & Diagnóstico sin resolver (A) & Diagnóstico incorrecto (B) & Error (A+B) & Precisión [\%] (100-Error) \\
\hline Nueva técnica & 0 & 4.1 & 4.1 & 95.9 \\
\hline Doernenburg & 15.1 & 1.1 & 16.1 & 83.9 \\
\hline Rogers & 37.6 & 17.2 & 54.8 & 45.2 \\
\hline IEC ratio & 17.2 & 16.1 & 33.3 & 66.7 \\
\hline Key gas & 3.2 & 38.7 & 41.9 & 58.1 \\
\hline
\end{tabular}

En el2013, Duval [20] propuso para el diagnóstico utilizar cuatro gases combustibles (hidrógeno, acetileno, etileno y metano) generados por averías internas en transformadores y analizar el porcentaje relativo de estos gases en función de los seis tipos de averías (PD, D1, D2, T1, T2 y T3); esto mediante dos técnicas: una consiste en distinguir entre averías basadas en el porcentaje relativo de esos cuatro gases, y la otra, en el uso de combinaciones del porcentaje relativo de dos de los cuatro gases.

La primera técnica utiliza el porcentaje relativo de los cuatro gases (Ecuación 4) para clasificar las zonas de acuerdo con los seis tipos de averías en un transformador, así: el porcentaje relativo de hidrógeno, correspondiente a averías eléctricas de baja energía; el porcentaje relativo de etileno, para averías de alta energía térmica; el porcentaje relativo de acetileno, por averías de energía eléctrica de alta, y el porcentaje relativo de metano, por averías térmicas de bajo consumo.

La Fig. 10 muestra los límites de la zona para cada tipo de avería.

$$
\begin{aligned}
& \% \boldsymbol{H}_{\mathbf{2}}=\mathbf{1 0 0} \frac{Z}{W+X+Y+Z} \\
& \% \boldsymbol{C H}_{\mathbf{4}}=\mathbf{1 0 0} \frac{Y}{W+X+Y+Z} \\
& \% \boldsymbol{C}_{\mathbf{2}} \boldsymbol{H}_{\mathbf{4}}=\mathbf{1 0 0} \frac{X}{W+X+Y+Z} \\
& \% \boldsymbol{C}_{\mathbf{2}} \boldsymbol{H}_{\mathbf{2}}=\mathbf{1 0 0} \frac{W}{W+X+Y+Z}
\end{aligned}
$$

Las zonas de avería eléctrica están situadas relativamente en la parte superior derecha, ya que producen gran parte de hidrógeno y acetileno, mientras las averías térmicas se distribuyen relativamente en la parte inferior izquierda debido a una gran parte de metano y etileno. 
La Fig. 11 muestra un diagrama de flujo para el diagnóstico de averías usando el porcentaje relativo de los cuatro gases, el cual se deduce a partir del cálculo del punto central donde se cruzan, y este proporciona un diagnóstico. A manera de ejemplo, si los porcentajes relativos son $30 \%$ de hidrógeno, $20 \%$ de etileno, $40 \%$ de acetileno y $10 \%$ de metano, respectivamente, el punto central es la zona en D2, de descarga de alta energía ( Fig. 12).

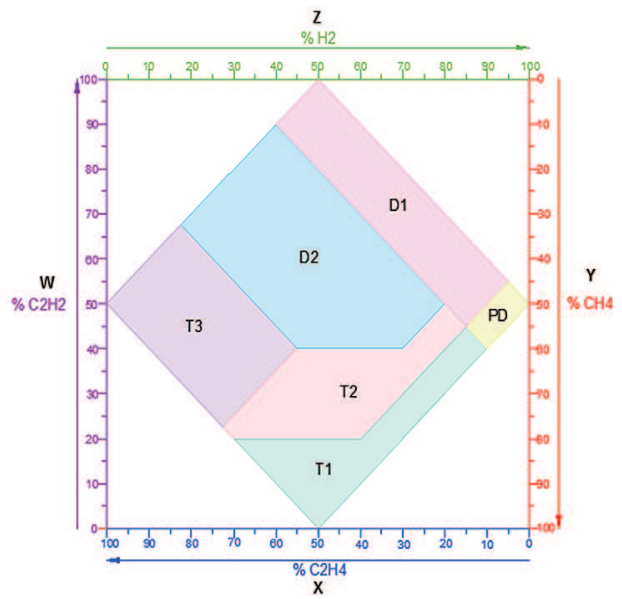

Fig. 10. Límites de zona para cada tipo de avería

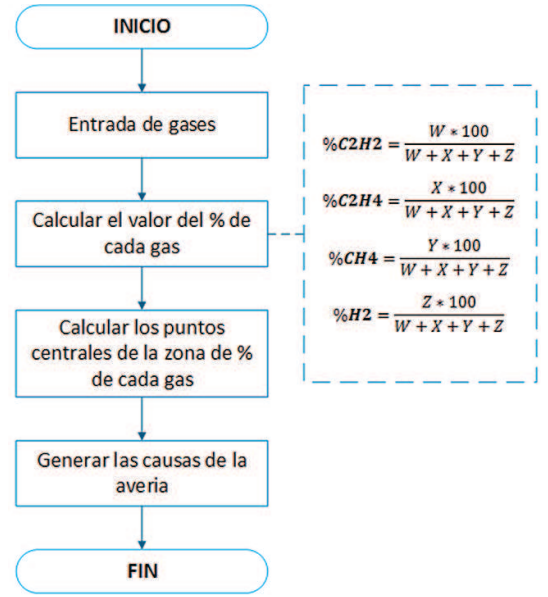

Fig. 11. Diagrama de flujo de la Técnica I

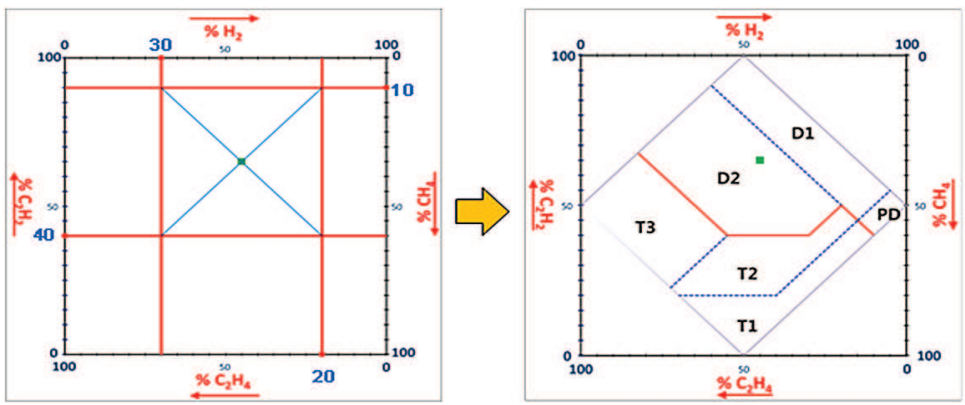

Fig. 12. Ejemplo de diagnóstico de averías D2.

La segunda técnica utiliza cuatro combinaciones del porcentaje relativo de dos gases y los tipos de avería detectables (Tabla 11). 
TABla 11

COMBINACIONES DE PORCENTAJE RELATIVO DE LOS GASES

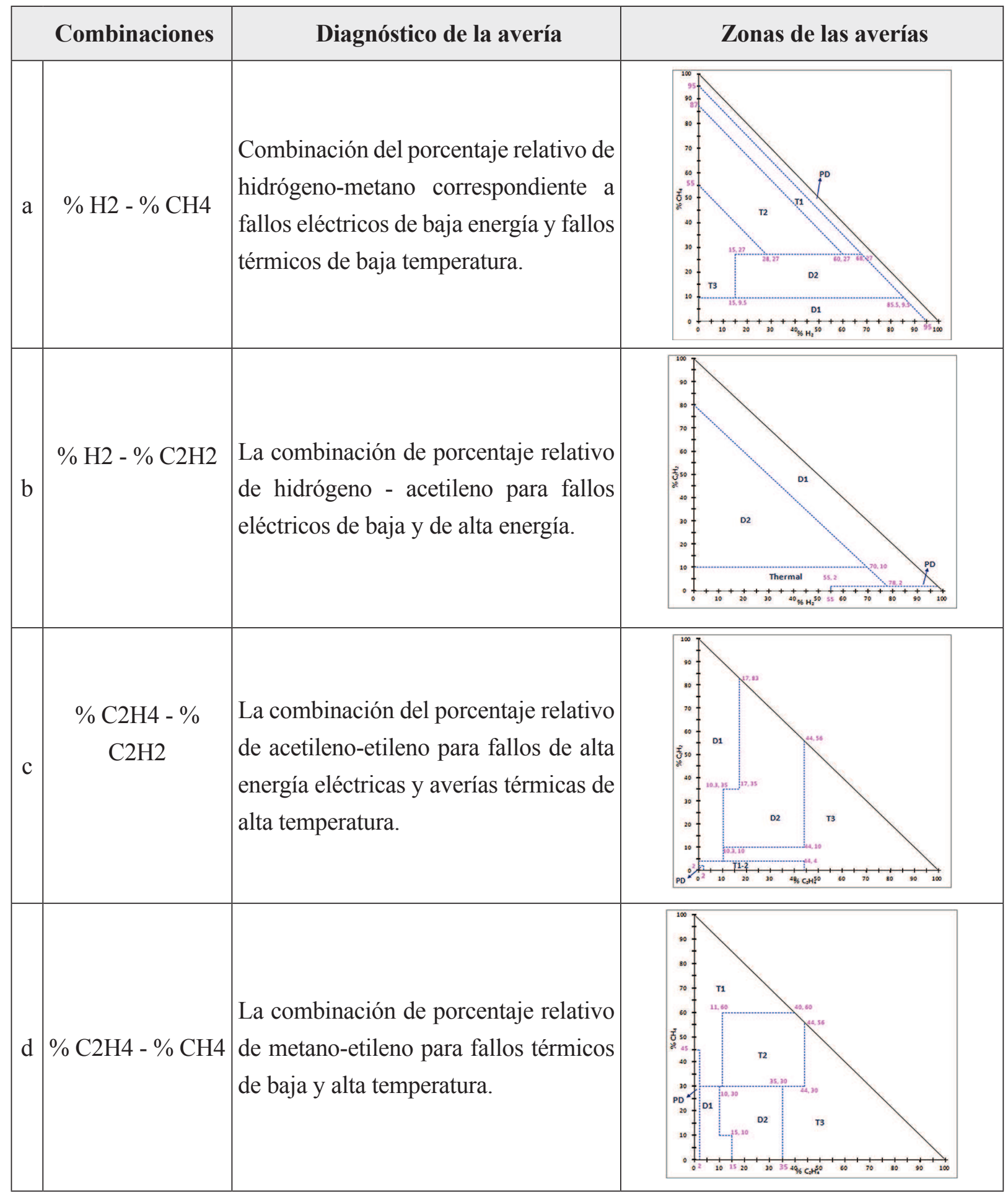

La Fig. 13 muestra el proceso de diagnóstico usando el porcentaje relativo de dos gases. 


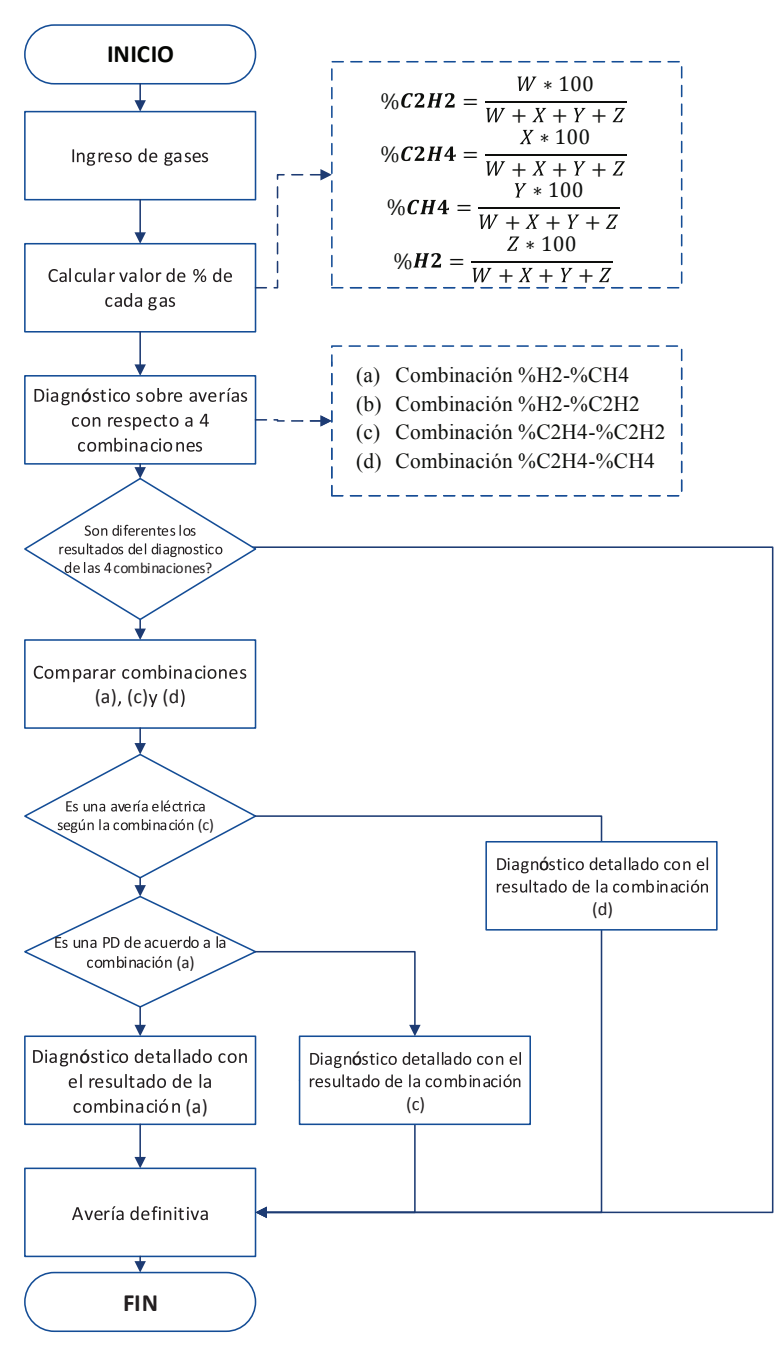

Fig. 13. Diagrama Técnica II

La Tabla 12 muestra que la técnica I y II de Duval tiene una precisión del 95\% y 96\%, respectivamente, en comparación con otras técnicas. La precisión fue calculada para los 122 casos de IEC TC 10 bases de datos.

\section{TABLA 12}

COMPARACIÓN ENTRE LA NUEVA TÉCNICA Y LAS TÉCNICAS EXISTENTES

\begin{tabular}{|c|c|c|c|c|}
\hline Técnica DGA & $\begin{array}{c}\text { Sin } \\
\text { resolver } \\
\text { (A) }\end{array}$ & $\begin{array}{c}\text { Mal } \\
\text { diagnóstico } \\
\text { (B) }\end{array}$ & $\begin{array}{c}\text { Error } \\
(\mathbf{A}+\mathbf{A})\end{array}$ & $\begin{array}{c}\text { Precisión } \\
\text { [\%] } \\
(\mathbf{1 0 0 - E r r o r )}\end{array}$ \\
\hline Técnica I & 0 & 4.6 & 4.6 & 95.4 \\
\hline Técnica II & 0 & 3.8 & 3.8 & 96.2 \\
\hline Doernenburg & 15.1 & 1.1 & 16.1 & 83.9 \\
\hline Rogers & 37.6 & 17.2 & 54.8 & 45.2 \\
\hline IEC & 17.2 & 16.1 & 33.3 & 66.7 \\
\hline Gases clave & 3.2 & 38.7 & 41.9 & 58.1 \\
\hline
\end{tabular}

Las dos técnicas de diagnóstico propuestas pueden realizar un análisis detallado de los seis tipos de averías internas (PD, D1, D2, T1, T2 y T3) definidos por la norma IEC 60599.

\section{Conclusiones}

Las técnicas de diagnóstico de DGA son complementarias, comúnmente utilizadas como guías para diagnosticar el estado de la condición del transformador; sin embargo, existe gran incertidumbre en los datos de los gases, debido a la variedad de patrones y a la cantidad de gases generados por los diferentes tipos de averías, que se encuentran afectadas por muchos factores, entre los cuales se destaca el tipo de aceite y su temperatura, el método de muestreo, las características de aislamiento y el medioambiente. Por lo tanto, con las diferentes técnicas de interpretación del DGA se pueden obtener interpretaciones de averías diferentes o en conflicto.

Una vez expuestas las diferentes técnicas de diagnóstico DGA, así como la precisión de cada una, se observa, al compararlas, que las nuevas técnicas de Duval presentadas este año tienen más del 95\% de precisión y son capaces de diagnosticar casi todo tipo de avería. 


\section{REFERENCIAS}

[1] M. Duval, «A Review of Faults Detectable by Gas-in-oil Analysis in Transformers,» IEEE Electrical Insulation Magazine, vol. $18, \mathrm{n}^{\mathrm{o}} 3,2002$.

[2] S. A. Ward, "Evaluating Transformer Condition Usiong DGA Oil Analysis," IEEE, vol. III, p. 6, 2003.

[3] M. Duval and J. Dukarm, "Improving the Reliability of Transformer Gas-in-Oil Diagnosis," IEEE, vol. 21, no. 4, pp. 21-27, 2005.

[4] J. Ganesha, Dissolved Gas Analysis of Transformer Oils: Effects of electric arc, Lisbon, Portugal: International Conference on Power Systems, September 22-24, 2006.

[5] M. Duval, «The Duval Triangle for Load Tap Changers, Non-Mineral Oils and Low Temperature Faults in Transformers,» IEEE, vol. 24, nº 6, pp. 22-29, 2008.

[6] A. S. H. B. E. G. A. Akbari, "A Software Implementation of the Dival Triangle Method," IEEE, p. 4, 2009.

[7] J. C. A. d. W. F. E. A. Mackenzie, "On-Line Monitoring and Diagnostics Form Power Transformers," IEEE, p. 5, 2010.

[8] S. S. S. a. M. A. V. Mohammad Golkhah, "Artificial neural networks applied to DGA for fault diagnosis in oil filled power transformers," Journal of Electrical and Electronics Engineering Research, vol. III, p. 10, 2011.

[9] Y.-C. H. C.-M. H. Huo-Ching Sun, "A Review of Dissolved Gas Analysis in Power Transformers," ELSEVIER, p. 6, 2012.
[10] D. Pugh, "Combustible Gas Analysis," Minutes of Fortieth International Conference of Doble Clients, pp. Section 10-401, 1973.

[11] D. Pugh, Advances in Fault Diagnosis by Combustible Gas Analysis, Minutes of Forty-First International Conference of Doble Clients, 1974, Section 10-1201 .

[12] M. Duval, "Dissolved Gas Analysis and the Duval Triangle,” Doble, pp. 1-20, 2006.

[13] M. Duval, "Dissolved Gas Analysis: It Can Save Your Transformer,” IEEE, p. 6, 1989.

[14] M. Duval, "Acceptable Gas-In-Oil Levels In Generation and Transmission Power Transformers," IEEE, p. 6, 1990.

[15] M. Duval and A. dePablo, "Interpretation of Gas-In-Oil Analysis Using New IEC Publication 60599 and IEC TC 10 Databases," IEEE, pp. 31-41, 2001.

[16] M.Duval, “Calculation ofDGALimit Values and Sampling Intervals in Transformers in Service," IEEE, vol. 24, no. 5, pp. 7-13, 2008.

[17] M. Duval, "The duval triangle for ltcs, alternative fluids and other applications," Doble, pp. 1-8, 2009.

[18] M. Duval, "New frontiers of DGA interpretation for power transformers and their accessories," SEEEI, pp. 1-8, 2012.

[19] M. Duval, w. K. Sung, j. K. Sung, d. S. Hwang, r. J. Jae and j. Y. Hang, "New Methods of DGA Diagnosis using IEC TC 10 and Related Databases Part 1: Application of Gas-ratio Combinations," IEEE, vol. 20, no. 2, pp. 1-8, 2013. 
[20] M. Duval, j. L. Soo, m. K. Young, d. S. Hwang, r. J. Jae and j. Y. Hang, "New Methods of DGA Diagnosis using IEC TC 10 and Related Databases Part 2: Application of Relative Content of Fault Gases," IEEE, vol. 20, no. 2, pp. 691-675, 2013.

[21] E. Rivas, Detección de averías en cambiadores de tomas en carga de transformadores basados en el patrón de vibraciones, Leganés: Universidad Carlos III de Madrid, 2009.

[22] J. MacDonal, Gas-in-oil Analysis as a Diagnostic Tool for Monitoring PowerTransformer Insulation Integrity, Minutes of Forty-Seventh International Conference of Doble Clients, 1980.

[23] ANSI/IEEE and C57.104-1991, C57.104 Guide for the Interpretation of Gases Generated in Oil Immersed Transformers, New York: Institute of Electrical and Electronic Engineers, 1994.

[24] S. Huo-Ching, H. Yann-Chang and H. ChaoMing, A Review of Dissolved Gas Analysis in Power Transformers, Taiwán(Republica de China): SciVerse ScienceDirect.

[25] V. D and T. S, Dissolved gas analysis of natural ester fluids under electrical and thermal stress, Alemania: Institute of Power Transmission and High Voltage Technology (IEH), University Stuttgart, 2008.

[26] L. Yishan, W. Zhenyuan and L. Yilu, An automated On-line monitoring and fault diagnosis system for power transformer, Canadá: IEEE, 2006.

[27] R. Naresh, V. Sharma and M. Vashis, An integrated neural fuzzy approach for fault diagnosis of transformers, India: IEEE, 2007.

[28] J. B. DiGiorgio, "Dissolved gas analysis of mineral oil insulating fluids," NTT Northern Technology and Testin, 2005.

[29] A. Rickley, A. Baker and J. G.W. Armstrong, "Analytical Techniques for Fault-Gas Analysis," Minutes of Forty-Fifth International Conference of Doble Clients, pp. Section 10-401, 1978.

[30] H. D. F. L. P. G. a. G. B. M. Duval, "Acceptable Gas-In-il Levels In Instrument Transformers (Current Vs Voltage Equipement)," IEEE, p. 6, 1991.

[31] M. Duval, "New Techniques for dissolved gas-in-oil analysis," IEEE, vol. 19, no. 2, pp. 6-15, 2003. 